\title{
Latent Autoimmune Diabetes in Adults (LADA): The Prevalent Form of Adult-Onset Autoimmune Diabetes in a Region of India
}

\author{
Alok Kumar1, 4,*, Alberto De Leiva ${ }^{1,2,3}$ \\ ${ }^{1}$ Department of Diabetes, Endocrinology and Nutrition, Hospital de Sant Pau, Universitat Autònoma de Barcelona (UAB), \\ Barcelona, Spain \\ ${ }^{2}$ Principal Investigator, EDUAB-IIB-HSP (CIBER-BBN, ISCIII), Universitat Autònoma de Barcelona (UAB), Spain \\ ${ }^{3}$ Scientific Director, Fundació Diabem, Barcelona, Spain \\ ${ }^{4}$ MK Diabetes Clinic, Ghaziabad, India
}

Received: August 22, 2017; Accepted: August 31, 2017; Published: September 15, 2017

*Corresponding author: Dr. Alok Kumar Postal address for communication: SB-132, Shastri Nagar, Ghaziabad-201002, India,

Tel: +919810943714;Email: dralokkr@hotmail.com

\section{Abstract}

Aims: We undertook a study to investigate the prevalence and general characteristics of patients with Latent autoimmune diabetes in adults (LADA).

Methods: This was a cross-sectional investigation conducted in the National Capital Region of India. Total of 139 subjects were recruited in the study. All subjects were screened for Glutamic acid decarboxylase autoantibody (GADA). GADA was analysed using ELISA kits with $98 \%$ specificity and $92 \%$ sensitivity.

Results: The prevalence of LADA was $6.5 \%$ (6.3\% in men and 6.8\% in women); 95\% Confidence interval (CI): $3.29-12.0 \%$ among adult-onset diabetic patients. Prevalence of LADA seemed to gradually decline with increasing age. LADA $(n=9)$ and DM2 $(n=130)$ patients were compared. LADA patients were younger $(p=0.045)$, had lower age at onset of diabetes $(p=0.025)$, waist circumference $(p=0.021)$. LADA patients had also longer duration of diabetes $(p=0.045)$. There was no significant difference in BMI between two groups.

Conclusions: Our results indicate that LADA represents $6.5 \%$ of cases among adult-onset diabetes in a region of north India. LADA is a prevalent form of adult-onset autoimmune diabetes and not rare. This study shows different phenotypic features of LADA patients compared to DM2 patients. In the developing world, sometimes routine antibody testing may be considered not to be cost-effective. Thus, clinical and phenotypic features of LADA may help clinicians recognize patients for potential antibody screening.

Keywords: Adult; Autoimmune; Diabetes; latent

Abbreviations: BMI : Body mass index; DASP : Diabetes antibody standardization program; DM1 : Type 1 diabetes; DM-1A : Classic Type 1 Diabetes; DM2 : Type 2 diabetes; ELISA : Enzymelinked immunosorbent assay; GADA : Glutamic decarboxylase autoantibodies; HLA : Human leukocyte antigen; IA-2A : Insulinomaassociated Antigen; ICA : Islet cell antibodies; IDS : Immunology of Diabetes society; LADA : Latent autoimmune diabetes in adults; TCF7L2 : Transcription factor 7-like 2; ZMIZ1 : Zinc finger MIZ-type containing $1 ; \mathrm{ZnT} 8 \mathrm{~A}$ : zinc transporter 8 autoantibodies

\section{Introduction}

Previously, autoimmune diabetes was generally considered to be a disease of childhood and adolescence, however, these days this concept does not hold true. It has been observed that adultonset autoimmune diabetes is not as rare as once considered. Almost, for last two decades, Latent autoimmune diabetes in adults (LADA) had been continually an area of interest and concern for researchers and clinicians worldwide. As the name suggests LADA is autoimmune mediated diabetes of adult onset defined by the presence of at least one diabetes associated pancreatic auto antibodies in their sera [1,2].

Glutamic decarboxylase autoantibodies (GADA) are the most prevalent and persistent autoantibodies in majority of patients with LADA. Although, the fluctuating titer of GADA in serum may pose challenges in the diagnosis of LADA in some patients $[3,4]$. Insulinoma-Associated Antigen (IA-2A) antibodies alone are not used to screen patients due to very low diagnostic sensitivity for LADA [5].

Preliminary data has shown that patients with autoimmune diabetes, characterized by the presence of GADA, display a different clinical phenotype from classical type 2 diabetes (DM2) without GADA [2,6,7].

Over the years, LADA has been extensively studied by various investigators but many controversial issues related to its etiopathogenesis and potential treatment options still prevail. Due to resemblance of phenotypic characteristics, slow onset and non requirement of insulin at diagnosis, LADA is generally misdiagnosed as DM2 and the correct diagnosis is delayed by years.

LADA has been described as a subtype of T cell-mediated DM-1 [3,8]. However, the association of DM-2 associated Transcription factor 7-like 2 (TCF7L2) and Zinc finger MIZ-type 
containing 1 (ZMIZ1) risk gene variants with LADA is important and against the arguments denying LADA as a distinct entity; also, the differences in human leukocyte antigen (HLA) haplotypes for both risk and protection complement the idea of LADA as a separate identity [9-13].

Both classic DM-1A and LADA are autoimmune in nature but lack of necessity of insulin treatment at diagnosis or at least within first six months after diagnosis clinically, differentiates LADA from classic DM1-A [14,15]. Mostly, only one pancreatic autoantibody is present in LADA patients whereas, DM-1A patients typically are positive for more than one pancreatic auto antibodies [16]. Epidemiological studies from different parts of the world have shown diverse prevalence of LADA ranging from $0 \%$ in an ethnic group of Papua New Guinea to approximately $10 \%$ in European individuals $[15,17]$.

In European Action LADA study, within the entire cohort of patients with autoimmune diabetes, $90.5 \%$ were positive for GADA. A significant difference was noted in the characteristics of autoantibodies-positive patients compared with autoantibodiesnegative patients. Action LADA experience has shown that nearly $10 \%$ of adult-onset diabetic patients have autoimmune diabetes, and LADA is more prevalent than classic type 1 diabetes [15].

Similarly, based on GADA positive results in a large LADA China study, 5.9\% patients were classified as LADA [18]. In concordance with European population, a clear difference in phenotypic and biochemical characteristics was observed in GADA-positive patients. In comparison with GADA-negative type 2 diabetic subjects, GADA positive subjects were leaner, had lower insulin secretion and less metabolic syndrome.

Data from South Asian region on LADA are sparse. Significant discrepancy in the frequency of LADA has been observed in earlier studies on Indian population ranging from $2.6 \%$ to $58 \%$ [19]. In part, such heterogeneity in the results could be attributed to the regional diversity and different methodology adopted by authors. Some of these studies included only specific subgroups like subjects with low BMI, young age $(<25$ years) and sulfonylurea failure with likelihood of high prevalence of LADA [19].

LADA may have ethnic variations and results from particular population cannot be extrapolated to other population. Diagnosing LADA early in the disease process is important as it may have therapeutic implications [19]. Many similarities and differences exist between Asian and European population with LADA [19]. To have a better understanding of LADA more research needs be carried out, especially in the developing economies with approximately $2 / 3$ of world's diabetic population [20]. In view of the extreme variations seen in the results of past studies on Indian subjects, we undertook a study to investigate the prevalence of LADA and also assess general characteristics of patients with LADA. To diagnose LADA, we adopted diagnostic criteria as suggested by Immunology of Diabetes society (IDS) and European Action LADA group using a Diabetes antibody standardization program (DASP) validated method.

\section{Methods}

\section{Subjects}

This study was conducted in the National Capital Region (NCR) of Northern India and included diabetic patients over 30 years of age consecutively attended at the Diabetes Clinic. Majority of the population investigated were inhabitants of the urban areas of NCR surrounding the capital city of New Delhi. All participants were natives of Northern states of India mainly Uttar Pradesh, Uttarakhand, Punjab, Haryana, Bihar and Delhi. The socio-economic status of most of the study participants ranged from lower middle to upper middle.

\section{Study Design}

This study was a cross-sectional investigation. The Sample size was calculated by accepting the confidence interval of $95 \%$ (0.95) for a precision of +/- 0.05 units in two sided test for an estimation proportion 0.1, from November 2015 until June 2016 total of 139 subjects were recruited in the study. Inclusion criteria were 1) age at diagnosis between $30-70$ years 2) Duration of disease between 6 months to 5 years. Any subject not fulfilling the inclusion criteria or not willing to participate were excluded.

In this population, subjects with glutamic acid decarboxylase autoantibody (GADA) in whom insulin was started at diagnosis or within 1 month after diagnosis were to be defined as DM1-A. [15] All antibody negative subjects were diagnosed as DM2. [21] LADA was defined as patients aged 30-70 years at the time of diabetes diagnosis who did not require insulin for at least 6 months after diagnosis, with GADA depicted at their sera.

\section{Methodology}

We reviewed detailed medical history of all subjects with the aim of collecting clinical and demographic data. All subjects were screened for GADA. GADA was analysed using RSR- ELISA kits (RSR Limited, Cardiff, UK) with 98\% specificity and 92\% sensitivity in the DASP 2005 [22]. Venous whole blood was collected in plastic serum separator tubes. Specimens were centrifuged for at least 15 minutes within one hour of collection. Thereafter, serum was transferred to properly labelled plastic-screw cap vial and frozen at -20 degree Celsius. All frozen samples were processed within a week of storage. Assay cut off of $<5 \mathrm{U} / \mathrm{ml}$ was considered negative and $\geq 5 \mathrm{U} / \mathrm{ml}$ was considered positive.

\section{Statistical analysis}

Quantitative variables were described as means \pm standard deviations or medians and inter quartile ranges. Categorical variables described were described as $\mathrm{n}$ (\%) Mean values between groups were compared using Independent t-Test/MannWhitney U test. The comparison of categorical variables was analyzed using chi-square /fisher exact test.

For all analyses, IBM SPSS statistics for windows software (version 21.0; Armonk NY, USA) and an alpha value of 0.05 for statistical significance were used. 


\section{Results}

\section{Demographics of study participants}

The 139 study subjects consisted of 80 (57.6\%) male and 59 $(42.5 \%)$ female. Overall, mean (SD) age was 46.7 (9.3) years and age at diagnosis was 43.9 (9.5) years. The mean disease duration was 34.5 (17.9), waist circumference was 96.3 (9.9) cms and BMI was $28.1(4.8) \mathrm{kg} / \mathrm{m} 2$. The demographics of studied participants are shown in (Table 1).

Table1: Baseline demographics of all 139 study subjects

\begin{tabular}{|c|c|}
\hline Characteristics & Mean \pm SD, Median $[I Q R]$ or $n(\%)$ \\
\hline Males, n (\%) & $80(57.6)$ \\
\hline Females, n (\%) & $59(42.4)$ \\
\hline Age (years) & $46.7 \pm 9.3$ \\
\hline Age at diagnosis (years) & $43.9 \pm 9.5$ \\
\hline $\begin{array}{l}\text { Duration of disease } \\
\text { (months) }\end{array}$ & $34.5 \pm 17.9$ \\
\hline Weight(kg) & $74.7 \pm 13.2$ \\
\hline $\mathrm{BMI}(\mathrm{kg} / \mathrm{m} 2)$ & $28.1 \pm 4.8$ \\
\hline Waist circumference $(\mathrm{cms})$ & $96.3 \pm 9.9$ \\
\hline $\begin{array}{l}\text { Family history of diabetes, } \\
\text { n (\%) }\end{array}$ & $90(64.7)$ \\
\hline \multicolumn{2}{|c|}{ Data are expressed as means (SD), median [IQR] or $n(\%)$} \\
\hline
\end{tabular}

\section{Prevalence of adult-onset autoimmune diabetes}

Within the entire cohort of 139 subjects with adult-onset diabetes, 9 (6.5\%) were positive for GADA. All antibody positive subjects did not require insulin within first six months after diagnosis of diabetes and were classified as LADA. As per the diagnostic criteria adopted by us, no subject was diagnosed with DM1-A.

GADA negative 130 subjects were diagnosed with DM2. The prevalence of LADA was $6.5 \%$ (6.3\% in men and $6.8 \%$ in women); 95\% Confidence interval (CI): $3.29-12.0 \%$ among adult-onset diabetic patients. Prevalence of LADA seemed to gradually decline with increasing age. However, the prevalence of DM2 continually increased with age. Age wise prevalence of LADA and DM2 is shown in (Table 2) and (Fig. 1).

\section{Comparison of phenotypic features of LADA and DM2 subjects}

LADA $(\mathrm{n}=9)$ and DM2 $(\mathrm{n}=130)$ patients were compared. LADA patients were younger $(40.8 \pm 7.6$ vs. $47.2 \pm 9.3$ years; $p=0.045)$, had lower age at onset of diabetes $(37.1 \pm 7.4$ vs. $44.4 \pm 9.4$ years; $\mathrm{p}=0.025)$, waist circumference $(88.9 \pm 8.3$ vs. $96.8 \pm 9.8 \mathrm{cms}$; $\mathrm{p}=0.021$ ). LADA patients had also longer duration of diabetes ( $46 \pm 12.7$ vs. $33.7 \pm 17.9 ; p=0.045$ ). Frequency of positive family history of diabetes was lower in LADA patients however, the difference was not statistically significant (44.4\% vs. 66.2 ; $\mathrm{p}=0.278$ ). There was no significant difference in BMI between two groups. The phenotypic characteristics of LADA and DM2 patients are summarized in (Table 3).
Table 2: Age and Gender wise prevalence of LADA and DM2 among all study subjects $n=139$

\begin{tabular}{|c|c|c|c|c|c|c|}
\hline \multirow{2}{*}{$\begin{array}{c}\text { Age } \\
\text { (years) }\end{array}$} & \multicolumn{3}{|c|}{ LADA } & \multicolumn{3}{c|}{ DM2 } \\
\hline \multirow{2}{*}{40} & Male & Female & Total & Male & Female & Total \\
\hline \multirow{2}{*}{$4 / 26$} & $15.4)$ & $1 / 10(10)$ & $\begin{array}{c}5 / 36 \\
(13.9)\end{array}$ & $\begin{array}{c}22 / 26 \\
(84.6)\end{array}$ & $\begin{array}{c}9 / 10 \\
(90)\end{array}$ & $\begin{array}{c}31 / 36 \\
(86.1)\end{array}$ \\
\hline $40-49$ & $1 / 33(3)$ & $\begin{array}{c}2 / 16 \\
(12.5)\end{array}$ & $3 / 49(6.1)$ & $\begin{array}{c}32 / 33 \\
(97)\end{array}$ & $\begin{array}{c}14 / 16 \\
(87.5)\end{array}$ & $\begin{array}{l}46 / 49 \\
(93.9)\end{array}$ \\
\hline $50-59$ & $0 / 18(0)$ & $1 / 22(4.5)$ & $1 / 40(2.5)$ & $\begin{array}{c}18 / 18 \\
(100)\end{array}$ & $\begin{array}{c}21 / 22 \\
(95.5)\end{array}$ & $\begin{array}{c}39 / 40 \\
(97.5)\end{array}$ \\
\hline$\geq 60$ & $0 / 3(0)$ & $0 / 11(0)$ & $0 / 14(0)$ & $3 / 3(100)$ & $\begin{array}{c}11 / 11 \\
(100)\end{array}$ & $\begin{array}{c}14 / 14 \\
(100)\end{array}$ \\
\hline Total & $5 / 80(6.3)$ & $4 / 59(6.8)$ & $9 / 139$ & $75 / 80$ & $55 / 59$ & $130 / 139$ \\
$(6.5)$ & $(93.8)$ & $(93.2)$ & $(93.5)$ \\
\hline
\end{tabular}

Data expressed as $n(\%)$

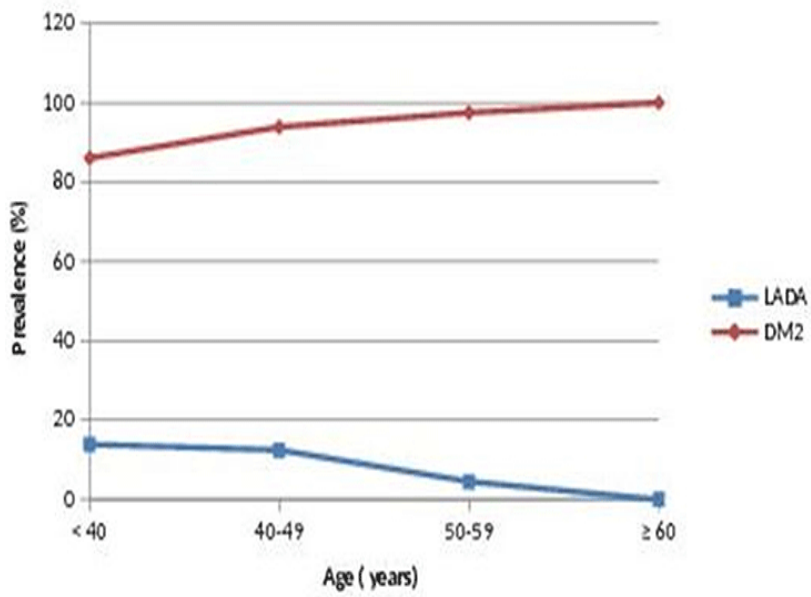

Figure 1: Age wise prevalence of LADA and DM2 among all study subjects.

Table 3: Characteristics of LADA subjects vs. DM2 subjects

\begin{tabular}{|c|c|c|c|}
\hline Cases & LADA ( $n=9)$ & DM2 $(n=130)$ & P-value \\
\hline Females, n (\%) & $4(44.4 \%)$ & $55(42.3 \%)$ & \\
\hline Males, n (\%) & $5(55.6 \%)$ & 75 (57.7\%) & 1 \\
\hline Age (years) & $40.8 \pm 7.6$ & $47.2 \pm 9.3$ & 0.045 \\
\hline $\begin{array}{l}\text { Age at diagnosis } \\
\text { (years) }\end{array}$ & $37.1 \pm 7.4$ & $44.4 \pm 9.4$ & 0.025 \\
\hline $\begin{array}{l}\text { Disease Duration } \\
\text { (months) }\end{array}$ & $46 \pm 12.7$ & $33.7 \pm 17.9$ & 0.045 \\
\hline BMI (Kg/m2) & $25.6 \pm 4.2$ & $28.3 \pm 4.8$ & 0.108 \\
\hline $\begin{array}{l}\text { Waist } \\
\text { circumference } \\
\text { (cms) }\end{array}$ & $88.9 \pm 8.3$ & $96.8 \pm 9.8$ & 0.021 \\
\hline $\begin{array}{l}\text { Family history of } \\
\text { Diabetes, n (\%) }\end{array}$ & $4(44.4 \%)$ & $86(66.2)$ & 0.278 \\
\hline
\end{tabular}

Data are expressed as means (SD), median [IQR] or $n(\%)$ 


\section{Discussion}

Adult-onset autoimmune diabetes had been continually an area of interest for clinicians and researchers worldwide. In the past, DM1-A was invariably considered to be the only form of autoimmune diabetes in both children and adults. However, in the last few decades growing number of publications have supported the idea of another form of adult- onset autoimmune diabetes called LADA, which in comparison to DM1-A, have slow onset, but generally show faster deterioration of $\beta$-cell function when compared to DM2. For many years, the concept of LADA as a distinct identity has faced many controversies [23]. The adaptation of age and insulin criteria's in defining LADA has been criticized [24,25].

In spite of all controversies, the concept about LADA, is undeniable today [2]. It may be known by any other name but clearly there is a sub-group of adult patients, clinically diagnosed as DM2 who are initially non insulin requiring and have evidence of autoimmunity. To some extent, neither age cut-off nor the time to insulin treatment means so much. However, from epidemiological point of view, to establish correct prevalence and characterize LADA in various populations, standardised diagnostic criteria is required. By adopting standardised criteria and valid methods, it would be more appropriate to do rational comparison between different populations.

Differences in the prevalence of LADA have been observed worldwide probably, due to the heterogeneity of type of adultonset autoimmune diabetes. Past studies involving Indian subjects have resulted in inconclusive heterogeneous information which can be attributed to the discrepancy in methodology adopted by various investigators to diagnose LADA $[26,27]$.

We investigated the prevalence of adult-onset autoimmune diabetes and characterized the phenotypic features of patients diagnosed with LADA in Asian Indians. The features of LADA were compared with antibody-negative patients with DM2.

In the current study, LADA emerged as the most prevalent form of adult-onset autoimmune diabetes. Similar observations of LADA being more prevalent than DM-1A were reported by a multicenter European Action-LADA study and a study from UAE involving a large cohort of adult-onset diabetic subjects $[15,28]$. As per Immunology of Diabetes society (IDS) and ActionLADA group diagnostic criteria, based on GADA measurement, the prevalence of LADA in our cohort was $6.5 \%$ which was surprisingly, exactly similar to the LADA prevalence reported in the northern region of China in a large multicenter LADA China study [18]. The overall prevalence of LADA in China, based on GADA determination, was 5.9\% [18].

The prevalence of LADA in our study was lower than 9.7\% and $11.6 \%$ observed in European subjects but higher than in a cohort from UAE $2.6 \%$, South Korea $1.7 \%$ and $4.4 \%$, Japan 3.8\% (EHIME study) and North America 4.2\% (ADOPT study) $[15,7,28,29,30,31,32]$. To ascertain prevalence of adult-onset autoimmune diabetes, similar to our study, some studies used only GADA whereas additionally IA-2/ICA and/or znt8 autoantibodies were used by others $[15,18,28,31]$.

GADA are most frequent and persistent autoantibodies [3]. Among GADA negative patients, only small proportion of patients are positive for other diabetes associated autoantibodies. Thus, majority of autoimmune diabetes in adults can be detected by using only GADA measurement and GADA only can be used for screening LADA specifically, in resource constraint settings in the developing world [3]. The expected difference in LADA prevalence using only GADA or GADA along with other diabetes autoantibody would not be significant [15]. In the past, low frequency of IA-2 autoantibody has been reported among adults of North Indian origin and Indo-Aryan children with DM1 [33,34].

Prevalence of LADA in our subjects was highest $13.9 \%$ in individuals aged $<40$ years; this is in contrast to the highest prevalence of $13.9 \%$ in patients aged 50-59 years in a small Chinese cohort [35]. However, consistent with our results, decreasing trend of LADA prevalence with increasing age was evident in large Chinese and European studies [15].

DM-1A was not prevalent in our cohort. There are no prevalence data available for adult-onset ( $>30$ years) DM-1A in North Indians but earlier studies have shown the prevalence of DM1-A to be lower among children and adolescents in North Indian populations than in Caucasians [34,36].

Frequency of GADA positivity in our cohort was considerably higher than $1.5 \%$ and $1.6 \%$ reported in two large studies involving adult north Indian subjects The participants of these two studies had higher mean age and longer duration of diabetes than in our study [33,37]. This can be the possible explanation for the discrepancy in results as diabetes autoantibodies in the sera tend to disappear with increasing age and duration of diabetes $[3,15]$. Moreover, sensitivity of the assay used for GADA measurement in one of these studies was lower than used in our analyses [37].

Prevalence of GADA positivity and LADA in the current study was much lower than demonstrated in Southern India $[27,38]$. This is contrary to the findings observed in China and Europe, where compared to north, the frequency of LADA was lower in the southern region [9,18,39-42]. Interestingly, lower frequency of LADA 2.6\% was noted in an island nation Srilanka near southern coast of India [43]. It needs to be assessed if geographical, climatic and cultural differences within a country or continent directly contribute towards regional discrepancies in the prevalence of LADA by conducting large scale studies.

Characteristics of LADA among European, Chinese and Arab populations are remarkably different from GADA-negative DM2 subjects $[15,18,28]$. In accordance, patients with LADA in our cohort, compared with DM2 patients, were younger at recruitment and onset of diabetes, had lower waist circumference.

There are some limitations of our study. We acknowledge that due to small sample size we cannot generalize our results however, to best of our knowledge this is the first study investigating the prevalence and general characteristics of LADA in the National Capital Region of India. Hence, our study paves the way for future research with large sample size. Further, due to 
inclusion of patients with disease duration up to five years after diagnosis, LADA cases might have been underestimated as GADA titer may fluctuate and decrease over time.[3]

\section{Conclusion}

Our results indicate that LADA represents $6.5 \%$ of cases among adult-onset diabetes in a region of north India. LADA is a prevalent form of adult-onset autoimmune diabetes and not rare. This study shows different phenotypic features of LADA patients compared to DM2 patients. These patients are generally misdiagnosed with DM2 and receive sulphonylurea therapy for years, which, further exhausts $\beta$-cells and aggravates the autoimmune process [44]. The knowledge that adult-onset diabetic have GADA should alert the health care providers to the high probability of more rapid progression to insulin therapy than in classic type 2 diabetes. In future, along with insulin, other potential pharmacological therapies like incretins, vitamin $\mathrm{D}$ and GAD65, which tend to preserve $\beta$-cells, may play an important role in the management of these patients [19].

To avoid use of $\beta$-cell damaging therapies and achieve optimal metabolic control in these patients it becomes increasingly important to screen for LADA early. Early insulin treatment in LADA may restore better metabolic control however; no clear potential long term effect has been demonstrated on preservation of beta cell function [45]. Not all, but majority of LADA patients tend to progress towards insulin dependency within a few years after the diagnosis. The risk of developing other organ-specific autoimmune diseases in LADA patients is high [5].

In the developing world, sometimes routine antibody testing may be considered not to be cost-effective. Thus, clinical and phenotypic features of LADA may help clinicians recognize patients for potential antibody screening.

\section{Acknowledgements}

"This work had been realized in the Medicine Doctorate framework of the Universitat Autònoma de Barcelona". Results of this study were presented in abstract form at the 26th American Association of Clinical Endocrinologists (AACE) annual scientific \& clinical congress, Austin, Texas, USA, May 3-7, 2017

\section{Human and animal rights}

Study was approved by the appropriate ethics committee and has been performed in accordance with the ethical standards laid down in the 1964 Declaration of Helsinki and its later amendments.

\section{References}

1. Tuomi T, Groop LC, Zimmet PZ, Rowley MJ, Knowles W, Mackay IR. Antibodies to glutamic acid decarboxylase reveal latent autoimmune diabetes mellitus in adults with a non-insulin-dependent onset of disease. Diabetes. 1993;42(2):359-362.

2. Zimmet PZ, Tuomi T, Mackay IR, Rowley MJ, Knowles W, Cohen M, et al. Latent autoimmune diabetes mellitus in adults (LADA): the role of antibodies to glutamic acid decarboxylase in diagnosis and prediction of insulin dependency. Diabet Med. 1994;11(3):299-303.
3. Huang G, Yin M, Xiang Y, Li X, Shen W, Luo S, et al. Persistence of glutamic acid decarboxylase antibody (GADA) is associated with clinical characteristics of latent autoimmune diabetes in adults: a prospective study with 3-year follow-up. Diabetes Metab Res Rev. 2016;32(6):615-622. Doi:10.1002/dmrr.2779.

4. ten Kate Q, Aanstoot HJ, Birnie E, Veeze H, Mul D. GADA persistence and diabetes classification. Lancet Diabetes Endocrinol. 2016;4(7):563564. Doi:10.1016/S2213-8587(16)30103-6.

5. Falorni A, Brozzetti A. Diabetes-related antibodies in adult diabetic patients. Best Pract Res Clin Endocrinol Metab. 2005;19(1):119-133. Doi:10.1016/j.beem.2004.11.010

6. Leslie RDG, Williams R, Pozzilli P. Clinical review: Type 1 diabetes and latent autoimmune diabetes in adults: one end of the rainbow. J Clin Endocrinol Metab. 2006;91(5):1654-1659. Doi:10.1210/jc.20051623

7. Turner R, Stratton I, Horton V, Manley S, Zimmet P, Mackay IR, et al. UKPDS 25: autoantibodies to islet-cell cytoplasm and glutamic acid decarboxylase for prediction of insulin requirement in type 2 diabetes. UK Prospective Diabetes Study Group. Lancet (London, England). 1997;350(9087):1288-1293.

8. Hawa MI, Buchan AP, Ola T, Wun CC, DeMicco DA, Bao W, et al. LADA and CARDS: a prospective study of clinical outcome in established adult-onset autoimmune diabetes. Diabetes Care. 2014;37(6):16431649. Doi:10.2337/dc13-2383

9. Zampetti S, Spoletini M, Petrone A, Capizzi M, Arpi ML, Tiberti C, et al. Association of TCF7L2 gene variants with low GAD autoantibody titre in LADA subjects (NIRAD Study 5). Diabet Med. 2010;27(6):701-704. Doi:10.1111/j.1464-5491.2010.02997.x

10.Andersen MK, Sterner M, Forsén T, Käräjämäki A, Rolandsson O, Forsblom C, et al. Type 2 diabetes susceptibility gene variants predispose to adult-onset autoimmune diabetes. Diabetologia. 2014;57(9):1859-1868. Doi:10.1007/s00125-014-3287-8

11. Rolandsson O, Palmer JP. Latent autoimmune diabetes in adults (LADA) is dead: long live autoimmune diabetes! Diabetologia. 2010;53(7):1250-1253. Doi:10.1007/s00125-010-1713-0

12. Luo S, Lin J, Xie Z, Xiang Y, Zheng P, Huang G, et al. HLA Genetic Discrepancy Between Latent Autoimmune Diabetes in Adults and Type 1 Diabetes: LADA China Study No. 6. 2016;101(4):1693-1700. Doi:10.1210/jc.2015-3771

13. Vatay A, Rajczy K, Pozsonyi E, Hosszúfalusi N, Prohászka Z, Füst G, et al. Differences in the genetic background of latent autoimmune diabetes in adults (LADA) and type 1 diabetes mellitus. Immunol Lett. 2002;84(2):109-115.

14. Naik RG, Brooks-Worrell BM, Palmer JP. Latent autoimmune diabetes in adults. J Clin Endocrinol Metab. 2009;94(12):4635-4644. Doi:10.1210/jc.2009-1120

15. Hawa MI, Kolb H, Schloot N, Beyan H, Paschou S a., Buzzetti R, et al. Adult-onset autoimmune diabetes in Europe is prevalent with a broad clinical phenotype: Action LADA 7. Diabetes Care. 2013;36(4):908913. Doi:10.2337/dc12-0931

16. Unger J. Latent Autoimmune Diabetes in Adults - American Family Physician. Am Fam Physician. 2010;81(7):843-847.

17. Dowse GK, Zimmet PZ, Spark RA, Mavo B, Rowley MJ, Mackay IR. Lack of antibodies to glutamic acid decarboxylase in young adults of the high diabetes prevalence Wanigela people of Papua New Guinea. Diabetes Res Clin Pract. 1994;24(3):195-198. 
18. Zhou Z, Xiang Y, Ji L, Jia W, Ning G, Huang G, et al. Frequency, immunogenetics, and clinical characteristics of latent autoimmune diabetes in China (LADA China Study): A nationwide, multicenter, clinic-based cross-sectional study. Diabetes. 2013;62(2):543-550. Doi:10.2337/db12-0207.

19. Kumar A, de Leiva A. Latent autoimmune diabetes in adults (LADA) in Asian and European populations. Diabetes Metab Res Rev. 2017;33(5):e2890. Doi:10.1002/dmrr.2890

20. International Diabetes Federation. IDF Diabetes. IDF Diabetes Atlas. 7th ed. Brussels, Belgium: International Diabetes Federation. 2015.

21. Alberti KG, Zimmet PZ. Definition, diagnosis and classification of diabetes mellitus and its complications. Part 1: diagnosis and classification of diabetes mellitus provisional report of a WHO consultation. Diabet Med. 1998;15(7):539-553. Doi:10.1002/ (SICI)1096-9136(199807)15:7<539::AID-DIA668>3.0.CO;2-S

22. Törn C, Mueller PW, Schlosser M, Bonifacio E, Bingley PJ. Participating Laboratories. Diabetes Antibody Standardization Program: evaluation of assays for autoantibodies to glutamic acid decarboxylase and islet antigen-2. Diabetologia. 2008;51(5):846-852. Doi:10.1007/s00125008-0967-2

23. Fourlanos S, Dotta F, Greenbaum CJ, Palmer JP, Rolandsson O, Colman PG, et al. Latent autoimmune diabetes in adults (LADA) should be less latent. Diabetologia. 2005;48(11):2206-2212. Doi:10.1007/s00125005-1960-7

24. Brophy S, Yderstraede K, Mauricio D, Hunter S, Hawa M, Pozzilli P, et al. Time to insulin initiation cannot be used in defining latent autoimmune diabetes in adults. Diabetes Care. 2008;3(3)1:439-41. Doi:10.2337/dc07-1308

25. Laugesen E, Østergaard JA, Leslie RDG. Latent autoimmune diabetes of the adult: current knowledge and uncertainty. Diabet Med. 2015;32(7):843-852. Doi:10.1111/dme.12700

26. Unnikrishnan AG, Singh SK, Sanjeevi CB. Prevalence of GAD65 Antibodies in Lean Subjects with Type 2 Diabetes. Ann N Y Acad Sci. 2004;1037:118-121. Doi:10.1196/annals.1337.018

27.Chandni R, Paul BJ, Udayabhaskaran V, Ramamoorthy KP. A study of non-obese diabetes mellitus in adults in a tertiary care hospital in Kerala, India. Int J Diabetes Dev Ctries. 2013;33:83-85. Doi: 10.1007/ s13410-013-0113-7

28. Maddaloni E, Lessan N, Al Tikriti A, Buzzetti R, Pozzilli P, Barakat MT, et al. Latent Autoimmune Diabetes in Adults in the United Arab Emirates: Clinical Features and Factors Related to Insulin-Requirement. PLoS One. 2015;10(8):e0131837. Doi:10.1371/journal.pone.0131837

29. Park YS, Wang CY, Ko KW, Yang SW, Park M, Yang MC, et al. Combinations of HLA DR and DQ molecules determine the susceptibility to insulin-dependent diabetes mellitus in Koreans. Hum Immunol. 1998;59(12):794-801.

30. Roh M-O, Jung C-H, Kim B-Y, Mok J-O, Kim C-H. The prevalence and characteristics of latent autoimmune diabetes in adults (LADA) and its relation with chronic complications in a clinical department of a university hospital in Korea. Acta Diabetol. 2013;50:129-134. Doi:10.1007/s00592-010-0228-y

31. Takeda H, Kawasaki E, Shimizu I, Konoue E, Fujiyama M, Murao S, et al. Clinical, autoimmune, and genetic characteristics of adult-onset diabetic patients with GAD autoantibodies in Japan (Ehime Study) Diabetes Care. 2002;25(6):995-1001.
32.Zinman B, Kahn SE, Haffner SM, O'Neill MC, Heise MA, Freed MI. Phenotypic Characteristics of GAD Antibody-Positive Recently Diagnosed Patients With Type 2 Diabetes in North America and Europe. Diabetes. 2004;53(12):3193-3200. Doi:10.2337/diabetes.53.12.3193

33. Britten AC, Jones K, Törn C, Hillman M, Ekholm B, Kumar S, et al. Latent autoimmune diabetes in adults in a South Asian population of the U.K. Diabetes Care. 2007;30:3088-3090. Doi:10.2337/dc07-0896

34. Kelly MA, Alvi NS, Croft NJ, Mijovic CH, Bottazzo GF, Barnett AH. Genetic and immunological characteristics of Type I diabetes mellitus in an Indo-Aryan population. Diabetologia. 2000;43(4):450-456. Doi:10.1007/s001250051328

35. Qi X, Sun J, Wang JJ, Wang PP, Xu Z, Murphy M, et al. Prevalence and correlates of latent autoimmune diabetes in adults in Tianjin, China: a population-based cross-sectional study. Diabetes Care. 2011;34(1):66-70. Doi: 10.2337/dc10-0488

36. Balasubramanian K, Dabadghao P, Bhatia V, Colman PG, Gellert SA, Bharadwaj U, et al. High Frequency of Type 1B (Idiopathic) Diabetes in North Indian Children With Recent-Onset Diabetes. Diabetes Care. 2003;26(9):2697. Doi:10.2337/diacare.26.9.2697

37. Sachan A, Zaidi G, Sahu RP, Agrawal S, Colman PG, Bhatia E. Low prevalence of latent autoimmune diabetes in adults in northern India. Diabet Med. 2015;32(6):810-813. Doi:10.1111/dme.12644

38. R.Anil Kumar, K.R.Narasimhasetty, H.R.S.Murthy, Suresh Somannavar, L.Murali, Punam V. Bhende, R.Lalitha SBS. GAD antibody positivity in South Indian Type2 Diabetic individuals. Int J Clin Cases Investig. 2013;5:92-98.

39. Radtke MA, Midthjell K, Nilsen TIL, Grill V. Heterogeneity of patients with latent autoimmune diabetes in adults: linkage to autoimmunity is apparent only in those with perceived need for insulin treatment: results from the Nord-Trøndelag Health (HUNT) study. Diabetes Care. 2009;32(2):245-250. Doi: 10.2337/dc08-1468

40.Tuomi T, Carlsson a, Li H, Isomaa B, Miettinen a, Nilsson A, et al. Clinical and genetic characteristics of type 2 diabetes with and without GAD antibodies. Diabetes. 1999;48(1):150-157. Doi:10.2337/ diabetes.48.1.150

41. Park Y, Hong S, Park L, Woo J, Baik S, Nam M, et al. LADA prevalence estimation and insulin dependency during follow-up. Diabetes Metab Res Rev. 2011;27(8):975-979. Doi:10.1002/dmrr.1278

42. Soriguer-Escofet F, Esteva I, Rojo-Martinez G, Ruiz de Adana S, Catalá M, Merelo MJ, et al. Prevalence of latent autoimmune diabetes of adults (LADA) in Southern Spain. Diabetes Res Clin Pract. 2002;56(3):213220.

43. Katulanda P, Shine B, Katulanda GW, Silva A, Asfir EL, Sheriff R, et al. Diabetes mellitus among young adults in Sri Lanka--role of GAD antibodies in classification and treatment: the Sri Lanka Young Diabetes study. Diabetologia. 2008;51(8):1368-1374. Doi:10.1007/ s00125-008-1052-6

44.Cabrera-Rode E, Perich P, Diaz-Horta O, Tiberti C, Molina G, Arranz C, et al. Slowly progressing type 1 diabetes: persistence of islet cell autoantibodies is related to glibenclamide treatment. Autoimmunity. 2002;35(7):469-474.

45. Thunander M, Thorgeirsson H, Torn C, Petersson C, Landin-Olsson M. $\beta$ - cell function and metabolic control in latent autoimmune diabetes in adults with early insulin versus conventional treatment: a 3-year follow-up. Eur J Endocrinol. 2011;164:239-245. Doi:10.1530/EJE-100901 